\title{
Piwi1 is essential for gametogenesis in mollusk Chlamys farreri
}

\author{
Xiaoshi Ma ${ }^{1}$, Aichang Ji ${ }^{1}$, Zhifeng Zhang ${ }^{1}$, Dandan Yang ${ }^{1}$, Shaoshuai Liang ${ }^{1}$, Yuhan Wang ${ }^{1}$, Zhenkui Qin \\ Corresp. 1 \\ ${ }^{1}$ Key Laboratory of Marine Genetics and Breeding (Ocean University of China), Ministry of Education, Ocean University of China, Qingdao, China \\ Corresponding Author: Zhenkui Qin \\ Email address: qinzk@ouc.edu.cn
}

Piwi (P-element induced wimpy testis) is an important gene involved in stem cell maintenance and gametogenesis in vertebrates. However, in most invertebrates, especially mollusks, the function of Piwi during gametogenesis remains largely unclear. To further understand the function of Piwi during gametogenesis, full-length cDNA of Piwi1 from scallop Chlamys farreri ( Cf-Piwil) was characterized, which consisted of a 2,637 bp open reading frame encoding an 878-amino acid protein. Cf-Piwi1 mRNA was mainly localized in the spermatogonia, spermatocytes, and oogonia, oocytes of early development and intra-gonadal somatic cells. Additionally, the knockdown of Cf-Piwil by injection of CfPiwi1-dsRNA (double-stranded RNA) into scallop adductor led to a loss of germ cells in C. farreri gonads. Apoptosis was observed mainly in spermatocytes and oocytes of early development, as well as a small number of spermatogonia and oogonia. Our findings indicate that Cf-Piwi1 is essential for gametogenesis in the scallop C. farreri. 


\section{Piwil is essential for gametogenesis in mollusk Chlamys}

2 farreri

3

4

5 Xiaoshi $\mathrm{Ma}^{1}$, Aichang Ji ${ }^{1}$, Zhifeng Zhang, Dandan Yang, Shaoshuai Liang, Yunhan Wang, and

6 Zhenkui Qin*

9 Key Laboratory of Marine Genetics and Breeding (Ocean University of China), Ministry of

10 Education, Qingdao, China

$13{ }^{1}$ These authors contributed equally to this work

$14 *$ Corresponding author.

15 E-mail address: qinzk@ouc.edu.cn

16 Tel./Fax: 0086-532-82032780 


\section{Abstract}

Piwi (P-element induced wimpy testis) is an important gene involved in stem cell maintenance and gametogenesis in vertebrates. However, in most invertebrates, especially mollusks, the function of Piwi during gametogenesis remains largely unclear. To further understand the function of Piwi during gametogenesis, full-length cDNA of Piwil from scallop Chlamys farreri (Cf-Piwil) was characterized, which consisted of a 2,637 bp open reading frame encoding an 878-amino acid protein. $C f$-Piwil mRNA was mainly localized in the spermatogonia, spermatocytes, and oogonia, oocytes of early development and intra-gonadal somatic cells. Additionally, the knockdown of $C f$-Piwil by injection of Cf-Piwil-dsRNA (double-stranded RNA) into scallop adductor led to a loss of germ cells in C. farreri gonads. Apoptosis was observed mainly in spermatocytes and oocytes of early development, as well as a small number of spermatogonia and oogonia. Our findings indicate that Cf-Piwil is essential for gametogenesis in the scallop C. farreri. 


\section{Introduction}

Gametogenesis is the basis of animal reproduction and mainly includes germ stem cell selfrenewal, meiosis and maturation of gametes. Studies of gametogenesis will help improve the reproductive ability and preservation of economically important species. It is known that many genes are involved in this process for model organisms, but the roles of these genes remain largely unknown in non-model organisms.

Piwi (P-element induced wimpy testis), a PIWI subfamily member of the Argonaute superfamily, is identified based on two conserved domains, PAZ and PIWI (Cerutti et al. 2000). The PAZ domain, at the center of the amino acid sequence, contains a typical single stranded nucleic acid binding motif that can bind to the 3' end of short RNA (Lingel et al. 2003; Yan et al. 2003). The PIWI domain, found in the C-terminal region, functions to maintain Piwi's stability and is structurally similar to the RNase H catalytic domain (Liu et al. 2004; Song et al. 2004).

The Piwi gene was first identified in Drosophila melanogaster and demonstrated a potentially important role in maintaining germ cells (GCs) (Lin and Spradling 1997). Subsequently, Piwi homologues were reported in a variety of species, including Caenorhabditis elegans, Bombyx mori, Danio rerio, Coturnix coturnix and Homo sapiens (Lau et al. 2001; Sasaki et al. 2003; Houwing et al. 2008; Chen et al. 2012; Tatsuke et al. 2014). The expression of the Piwi gene is mostly restricted to gametogenesis and early embryonic development, but its expression pattern and functions are not consistent in different animals (Deng and Lin 2002; Megosh et al. 2006; Carmell et al. 2007; Houwing et al. 2008; Wang and Reinke 2008). In D. melanogaster, Piwi mutants eliminate the self-renewing division of germ stem cells (GSCs), and overexpressing Piwi in the germarium somatic cells results in an increase in number of GSCs and the rate of mitosis (Cox et al. 1998). In the flatworm Macrostomum lignano, knockdown of Piwi results in a complete elimination of all stem cells, including GSCs and somatic stem cells (De Mulder et al. 2009). Tatsuke et al. (2014) suggested that Siwi (the silkworm homologue of the Piwi protein) recruits HP1 proteins to a target site guided by the Piwi-piRNA complex, and then the Piwi-HP1 complex functions as a rapid transcriptional repressor to regulate gene 
expression in B. mori.

Mollusks are one of the most abundant and biologically diverse groups in the animal kingdom. Identifying early GCs is beneficial to the study of gametogenesis. However, it is difficult in histological sections of the mollusk gonads to distinguish various types of germ cells accurately, especially for the early development stages, because some features, such as cell size and karyoplasmic ratio, are diverse in different sections. These problems limit the study on the molecular mechanism of gametogenesis in mollusk. In this study, we cloned full-length cDNA of Piwil in the scallop Chlamys farreri, a commercially important bivalve mollusk in China, and revealed its expression characteristics in the gonads during gametogenesis. Furthermore, the role of C. farreri Piwil (Cf-Piwil) in the scallop during gametogenesis was examined using RNAi. Our aims are to demonstrate the function of $C f$-Piwil during gametogenesis and investigate its potential feasibility as a molecular marker to identify early GCs in the scallop gonads.

\section{Materials and methods}

\section{Ethics Statement}

The collection and handing of the scallops $C$. farreri were performed in accordance with the Institutional Animal Care and Use Committee of the Ocean University of China and the local government.

\section{Specimen collection and sampling}

Adult $C$. farreri scallops with a mean shell height of $6.28 \pm 0.43 \mathrm{~cm}$ were collected from Shazikou (Qingdao, China). Gonads were dissected into $0.2 \mathrm{~cm}^{3}$ pieces. Some of these pieces were fixed in $4 \%$ paraformaldehyde in $0.1 \mathrm{M}$ phosphate buffer $(\mathrm{pH} 7.4)$ at $4^{\circ} \mathrm{C}$ for $24 \mathrm{~h}$, dehydrated through serial methanol dilutions $(25,50,75$ and 100\%) and stored in pure methanol at $-20^{\circ} \mathrm{C}$ for in situ hybridization (ISH). Some other pieces were fixed in Bouin's solution (picric acid, saturated aqueous solution - $75 \mathrm{ml}$; formalin, 40\% aqueous solution - $25 \mathrm{ml}$; acetic acid, glacial - $5 \mathrm{ml}$ ) for $24 \mathrm{~h}$ and then stored in $70 \%$ ethanol for histological observation. The 
82

remaining pieces were immediately frozen in liquid nitrogen and stored at $-80^{\circ} \mathrm{C}$ for total RNA isolation. All the reagents used without specific indication were provided by Sangon Biotech (Shanghai, China).

\section{Histology}

Gonads stored in $70 \%$ ethanol were dehydrated in an ethanol dilution series, cleared with xylene, and embedded in paraffin wax according to the description of Liu et al. (2014). Sections were made at $5 \mu \mathrm{m}$ thickness and stained with hematoxylin and eosin. Observations and digital images were taken with a Nikon E80i microscope (Nikon, Tokyo, Japan).

Gonads were divided into four stages according to previously described morphological characteristics (Liu et al. 2012). The gonadosomatic indices (GSI = gonad weight/soft tissue body weight $\times 100 \%$ ) are defined as resting stage (GSI 3.73\% for females and 3.49\% for males), proliferative stage (GSI 4.32\% for females and $4.38 \%$ for males), growing stage (GSI 5.39\% for females and $5.42 \%$ for males) and mature stage (GSI $14.29 \%$ for females and $12.48 \%$ for males).

\section{Total RNA extraction and reverse transcription}

Total RNA was extracted using the thiocyanate-phenol-chloroform method according to Chomczynski et al (1987) . Quality and quantity of the RNA were measured using agarose gel electrophoresis and spectrophotometry. Reverse transcription for full-length cDNA cloning and qRT-PCR were performed according to manufacturer instructions using the SMARTer ${ }^{\mathrm{TM}}$ RACE cDNA Amplification Kit (Clontech, Mountain View, USA) and Primescript ${ }^{\mathrm{TM}} \mathrm{RT}$ reagent Kit with gDNA Eraser (Perfect Real Time) (Takara, Otsu, Japan), respectively.

\section{Cloning and sequence analysis of full-length cDNA}

A Piwil cDNA fragment of 311 bp was obtained from the $C$. farreri transcriptome (Wang et al., 2013) and compared to the National Center for Biotechnology Information (NCBI) database using BLASTX. Amplification of 5'- and 3'-RACE were conducted with scallop testis cDNA and two specific PCR primers (PR-5': 5'-GCAACAGACATCAACATCTGTTTCTTGG-3', PR- 
107

108

109

110

111

112

113

114

115

116

117

118

119

120

121

122

123

124

125

126

127

128

129

130

131

132

133

3': 5'-ATGCTGATTGGAGCAGAGATCTTCGTGG-3') according to the SMART ${ }^{\text {TM }}$ RACE cDNA Amplification Kit protocol (Clontech, Mountain View, USA). PCR products were gelpurified and cloned into the pMD18-T vector (Takara, Otsu, Japan) then transformed into Escherichia coli DH5 $\alpha$ competent cells (Takara, Otsu, Japan). Positive clones were selected and sequenced. The full-length cDNA sequence was assembled using DNASTAR, Lasergene version 7.1 .

The identity and similarity of the deduced amino acid sequence were analyzed with other known PIWI1 (Homo sapiens, Mus musculus, Sus scrofa, Gallus gallus, Caprimulgus carolinensis, Xenopus tropicalis, Danio rerio, Alitta virens, Lottia gigantea, Crossostrea gigas, Mytilus galloprovincialis, Caenorhabditis elegans) in GenBank using the online BLASTX tool.

Multiple alignments were performed using the software CLUSTALX version 1.81 and DNAMAN version 8.0. We conducted a phylogenetic analysis using the neighbor-joining method in MEGA 5.0 with 1,000 bootstrap replicates (Koichiro et al. 2011).

\section{qRT-PCR analysis}

qRT-PCR was conducted using SYBR Green Real-Time PCR Master Mix (TOYOBO, Osaka, Japan) and an ABI 7500 Real-Time PCR System (Applied Biosystems, Foster City, USA). A parallel amplification of the $C$. farreri elongation factor $1 \alpha(E F-1 \alpha)$ reference transcript (GenBank accession no. AEX08674.1) was performed to normalize expression data of Cf-Piwil transcripts. Two pairs of specific primers, Piwi 1F-1: 5'-CGGAGGCGTTGTGTGTAGCA-3', PiwilR-1: 5'- CTGTCCATCCCCAACACCATC-3' for amplifying a 193 bp of Cf-Piwil, and

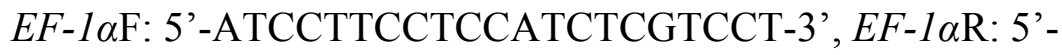

GGCACAGTTCCAATACCTCCA-3' for amplifying an 86 bp of $C f-E F-1 \alpha$ were designed. RTPCR conditions consisted of an initial denaturation step at $95^{\circ} \mathrm{C}$ for $30 \mathrm{~s}$ followed by 40 cycles of $5 \mathrm{~s}$ of denaturation at $95^{\circ} \mathrm{C}$ and $30 \mathrm{~s}$ of annealing and extension at $60^{\circ} \mathrm{C}$. Gonads from five individuals at each developmental stage were sampled, and triplicate assays for each gonad cDNA were conducted. The data were analyzed using the ABI 7500 system SDS software version 1.4 with automatically set baseline and cycle threshold values. Relative Cf-Piwil mRNA 
134

135

136

137

138

139

140

141

142

143

144

145

146

147

148

149

150

151

152

153

154

155

156

157

158

159

levels were calculated based on the $2^{-\Delta \Delta \mathrm{Ct}}$ method.

All data are presented as the means \pm standard error of five samples with three parallel repetitions. Differences between the means were tested using one-way analysis of variance (ANOVA) followed by least significant difference tests with the significance level set at $P<0.05$ in SPSS version 17.0.

\section{Tissue ISH}

DIG-labeled RNA sense and anti-sense probes were synthesized from a $557 \mathrm{bp}$ fragment of Cf-Piwil from sites 3078 to 3634 according to instructions with the DIG RNA Labeling kit

(Roche, Basel, Switzerland). Gonads stored for tissue ISH were cleared in xylene and embedded in paraffin wax before sectioning at $5 \mu \mathrm{m}$ for testes and $7 \mu \mathrm{m}$ for ovaries. Sections were fixed to a slide with $0.1 \%$ polylysine for $10 \mathrm{~h}$ at $37^{\circ} \mathrm{C}$. Before washing three times with PBST (phosphate-buffered saline with $0.1 \%$ Tween 20) and digesting with proteinase $\mathrm{K}\left(2 \mu \mathrm{g} \mathrm{ml}^{-1}\right)$ for $15 \mathrm{~min}$ at $37^{\circ} \mathrm{C}$, samples were dewaxed in xylene and rehydrated through a descending series of methanol dilutions. After fixing with $4 \%$ paraformaldehyde for $1 \mathrm{~h}$ and prehybridizing at $60^{\circ} \mathrm{C}$ for $6 \mathrm{~h}$ in hybridization buffer (50\% formamide, 5\% SSC, $5 \mathrm{mM} \mathrm{EDTA,} 100 \mathrm{mg} \mathrm{ml}^{-1}$ ribonucleic acid, $1.5 \%$ blocking reagent, $0.1 \%$ Tween 20 ), samples were hybridized with digoxigenin (DIG)labeled probes at $1 \mathrm{mg} \mathrm{ml}^{-1}$ in hybridization buffer for $16 \mathrm{~h}$ at $60^{\circ} \mathrm{C}$. Following hybridization, samples were washed in maleic acid buffer $(0.1 \mathrm{M}$ maleic acid, $0.15 \mathrm{M} \mathrm{NaCl}, 0.1 \%$ Tween 20 , $\mathrm{pH} 7.5$ ) and incubated with alkaline phosphatase-conjugated anti-DIG antibody from DIG Nucleic Acid Detection Kit (Roche, Basel, Switzerland) at $4^{\circ} \mathrm{C}$. After staining with NBT/BCIP (Roche, Basel, Switzerland) for $3 \mathrm{~h}$ at room temperature, the sections were counterstained with 1\% neutral red. Hybridization signals were detected and photographed using a Nikon E80i microscope (Nikon, Tokyo, Japan).

\section{dsRNA synthesis}

A 726 bp fragment of $C f$-Piwil cDNA from sites 11 to 736 was amplified using the primers PiwilF-2: 5' -TAATACGACTCACTATAGGGTTGAGAGGCAAGAAGTAACA-3' and 


\section{PiwilR-2: 5'-TAATACGACTCACTATAGGGGTACAGATGAAGGCACTGTG-3’ (T7} promoter sequence underlined) with $C$. farreri testis RNA as the template. The purified PCR fragment was transcribed, and the double-strand RNA (dsRNA) was synthesized in vitro using T7 MEGAscript RNAi Kits (Ambion, Austin, USA) according to manufacturer instructions. Quality and quantity of the Piwil-dsRNA was measured by 1\% agarose gel electrophoresis and spectrophotometry.

\section{dsRNA injection and sampling}

Scallops with a mean shell height of $6.13 \pm 0.54 \mathrm{~cm}$ at the proliferative stage were collected from Shazikou (Qingdao, China) and maintained in aerated running filtered seawater and fed with single cell algae during the experiment. A total of 75 scallops were randomly assigned to 3 groups. Scallops from the dsRNA group and PBS group were injected with $25 \mu \mathrm{g} C f$-Piwi 1 dsRNA diluted in $100 \mu \mathrm{l}$ PBS and $100 \mu \mathrm{PBS}$ only into adductor muscle, respectively. Scallops in the blank group were not injected with anything. Two injections were conducted during the experiment, at the beginning and at day 7 of the experiment. On the 3 rd day after injection, 3 scallops from each group were removed randomly, and their gonads were sampled as described above to estimate the $C f$-Piwil knockdown effect. On the $10^{\text {th }}$ day, 8 scallops ( 5 males and 3 females) from each group were sampled again.

\section{Types of germ cells quantification}

Five- $\mu \mathrm{m}$ gonadal sections were conducted from scallops of each group following the method mentioned in Histology. To determine the effect of Cf-Piwil knockdown, five squares (6400 $\mu^{2}$ for ovary and $1600 \mu \mathrm{m}^{2}$ for testis) of the sections were randomly picked to calculate the mean number and composition of germ cells in the gonads of each group. Differences between cell quantities of different groups were tested using one-way analysis of variance (ANOVA) followed by least significant difference tests with the significance level set at $P<0.05$ 
in SPSS version 17.0.

\section{TUNEL assay}

Five- $\mu \mathrm{m}$ sections of the gonads were prepared after the RNAi experiment. To detect in situ cell apoptosis, a TUNEL (terminal deoxynucleotidyl transferase-mediated dUTP nick end labeling) assay was performed using a TdT-mediated dUTP apoptosis detection kit (Promega, Madison, USA) with a hematoxylin counterstain. The sections were observed and photographed using a Nikon E80i microscope (Nikon, Tokyo, Japan).

\section{Results}

\section{Sequence and characteristics of the $C f$-Piwil full-length cDNA}

The full length of the Cf-Piwil cDNA was 4,986 bp (GenBank accession number: KR869093) with a 59 bp 5' untranslated region (UTR), a 2,290 bp 3' UTR and a 2,637 bp open reading frame (ORF), encoding a putative protein of 879 amino acids, with a predicted molecular weight of $99.34 \mathrm{kDa}$ and theoretical isoelectric point of 9.16. Multiple alignment indicated that the predicted protein contained a PAZ domain and a PIWI domain (Fig. S1) and was highly homologous to other known PIWI1, with 57\% identity to Crassostrea gigas, 51\% to Danio rerio and $53 \%$ to Homo sapiens. Phylogenetic analysis showed that the predicted $C f$-Piwil first clustered with C. gigas and M. galloprovincialis, and then followed established evolutionary hypotheses (Fig. 1).

\section{Quantitative expression of $C$ f-Piwi1 $\mathrm{mRNA}$ in $C$. farreri gonads during the reproductive cycle}

Expression levels of $C f$-Piwil in C. farreri gonads increased significantly from the resting to the mature stage (Fig. $2 \mathrm{I}$ and II ). Cf-Piwil expression in testes at the mature stage was approximately 2.5 times higher than that of the resting stage. In ovaries, it was about two-fold higher at the mature stage than the resting stage. No significant differences in Cf-Piwil 
208

209

210

211

212

213

214

215

216

217

218

219

220

221

222

223

224

225

226

227

228

229

230

231

232

expression levels were observed between the ovary and testis at the same developmental stages $(P>0.05)$.

\section{Cytolocation of $C f$-Piwil mRNA in $C$. farreri gonads during gametogenesis}

Cf-Piwil mRNA was mainly located in GCs during early development. In ovaries, Cf-Piwi1 transcripts were detected in oogonia, oocytes of early development and intra-gonadal somatic cells (ISCs) of germinal acini, but no positive signal was detected in mature oocytes (Fig. 2II ad). In testes, obvious positive signals were observed in spermatogonia, spermatocytes and ISCs of germinal acini. However, no positive signal was visible in spermatids and spermatozoa (Fig. $2 \mathrm{III}$ e-i). Moreover, no positive signal was detected in gonads using sense probes (Fig. $2 \mathrm{III}$ b0, $\mathrm{d} 0, \mathrm{~g} 0)$.

\section{Cf-Piwil knockdown led to abnormal development and apoptosis of GCs}

qRT-PCR detected that levels of the Cf-Piwil mRNA decreased significantly in the gonads of the dsRNA group than that of the PBS and Blank groups. The reduction of Cf-Piwil expression levels in the $C$. farreri gonads between the first injection and the second injection in the dsRNA group was very similar, and the declines in the ovaries and testes were approximately $30 \%$ or $35 \%$ of that in the blank group, respectively (Fig. 3 I and II ).

To investigate the effects of Cf-Piwil deficiency on gametogenesis, we performed a histological analysis. Cf-Piwil-dsRNA provoked several defects in development of GCs in both testes and ovaries. Compared with scallops from control groups, most oocytes in the ovaries of dsRNA scallops were at early developmental stage and many of them were stained darkly and presented abnormal morphological characteristics, implying that Cf-Piwil downregulation might inhibit oocyte development (Fig. 3 III a-f). In the testes of the Cf-Piwil knockdown scallops, the arrangement of GCs in the germinal acini became loose, spermatids occurred only in few germinal acini and the number of spermatids was smaller compared visually to that of the blank and PBS groups (Fig. 3 III g-1). Furthermore, we quantified the number and composition of germ 
233

234

235

236

237

238

239

cells in each group. In ovaries, more than half number of all kinds of germ cells decreased after knocking down of Cf-Piwil, but the proportion of Cf-Piwil expression cells (ISC 36.3\%, oogonia $30.5 \%$ and oocyte $33.2 \%$ ) increased when comparing with control groups (ISC 32.1\%, oogonia $35.7 \%$ and oocyte 18.2\%) (Fig. 4 I , Table S1). Similar results were obtained in testis with the percentage of spermatogonia and spermatocyte changed from $11.4 \%$ and $67.2 \%$ of the control groups to that of $38.2 \%$ and $54.5 \%$ after RNAi (Fig. 4 II, Table S2). Interestingly, we also found in some germinal acini of the Cf-Piwil knockdown scallops, number of spermatocytes greatly decreased while spermatogonia and spermatids persisted (Fig. 3 III i\&l).

The TUNEL assay results revealed that some of the oogonia and many oocytes were in the process of apoptosis in the ovaries of the dsRNA scallops, and the majority of spermatocytes and partial spermatogonia in Cf-Piwil knockdown testes were undergoing apoptosis (Fig. $4 \mathrm{III} \mathrm{c}, \mathrm{f}, \mathrm{i}$, 1). Few apoptotic cells were found in the gonads of the blank and PBS groups (Fig. 4III a, b, d, e, $\mathrm{g}, \mathrm{h}, \mathrm{j}, \mathrm{k})$.

\section{Discussion}

\section{Cf-Piwil expression pattern in GCs is similar to that of fish}

Localizations of Piwi transcripts are diverse in gonads of different species, although they are known to express mainly in GCs. In the planarian Schmidtea mediterranea, Piwi mRNA is visible in somatic stem cells and GCs (Reddien et al. 2005; Rossi et al. 2006; Palakodeti et al. 2008). In D. melanogaster, Piwi is expressed in all the cells of gonads (Cox et al. 2000). In D. renio, Ziwi, a Piwi homologue, is found only in GCs of gonads, where its expression appears to be the strongest in GCs at the mitotic and early meiotic stages (Houwing et al. 2008). Similarly, in medaka (Oryzias latipes), Piwi is expressed in spermatogonia, spermatocytes and all ovarian GCs (Li et al. 2012). In M. musculus, Miwi expression appears to be restricted to the primary spermatocytes, secondary spermatocytes and the elongating spermatids, and no expression is observed in somatic cells of testis (Deng and Lin 2002). In this study, 
257

258

259

260

261

262

263

264

265

266

267

268

269

270

271

272

273

274

275

276

277

278

279

280

281

282

Cf-Piwil mRNA was expressed in male and female GCs of early development, which differs from that in mammals but is similar to that in fish.

\section{Cf-Piwil is possibly a molecular marker for early GCs}

Yano et al. (2008) reported that rtili, a homolog of Piwi in the rainbow trout Oncorhynchus mykiss, is expressed specifically in spermatogonia, and is used as a molecular marker to identify spermatogonia. In this study, we found that Cf-Piwil expression was not only restricted to spermatogonia, but was also specifically visible in the GCs of early development, such as spermatogonia, spermatocytes, oogonia, and oocytes of early development. Thus, it can be potentially used to identify the GCs of early development in the testes and ovaries of $C$. farreri.

\section{Cf-Piwil is essential for gametogenesis in C. farreri}

In model animals, the roles of Piwi on gametogenesis are diverse, but Piwi defects always result in the loss of GCs, reductions in nurse cell, poorly-developed egg chambers, and complete female sterility (Lin and Spradling 1997). Cox et al. (1998) reported that Piwi mutations in D. melanogaster cause loss of GCs, but no dead cells were detected, which implies that the loss of Piwi can eliminate the self-renewing division of GSCs. Similarly, in C. elegans, decreasing Piwi expression by RNAi reduces the proliferation of GSC-equivalent cells (Cox et al. 1998). Moreover, in Zili (Ziwi-like) mutant zebrafish, almost all GCs are lost yet no apoptosis is present, suggesting that loss is possibly due to their inability to proliferate and differentiate (Houwing et al. 2008). Mutation of a hypomorphic Zili allele blocks oogenesis in Meiosis I and induces terminal female sterility (Houwing et al. 2008). However, Houwing et al. (2007) found that the reduction of Ziwi in D. renio leads to various spermatogenic cell losses by apoptosis. In M. musculus, significant numbers of apoptotic cells were detected in spermatocyte layers due to the loss of Mili (Kuramochi-Miyagawa et al. 2004). Miwi-knockout mice display a drastic increase in apoptotic cell numbers of testes and spermatogenic arrest at the round spermatid stage (Deng and Lin 2002). Additionally, Miwi2 mutants exhibit spermatogenic cell apoptosis and predominantly arrest at the leptotene stage of meiosis (Carmell et al. 2007).

In this study, we found that knockdown of $C f$-piwil lead to significant cell number reduction and 
283

284

285

286

287

288

289

290

291

292

293

294

295

296

297

298

299

300

301

302

303

304

305

306

307

308

most of the remaining germ cells detained at the early development stages, implying its important role in the germ cell proliferation and differentiation. In the meantime, the proportion Cf-piwil expression cells increased after RNA interference also indicated the gametogenic arrest occurred at early development stages, which was accordant with the reported studies. Cell apoptosis assay presented that marked apoptosis occurred mainly in the spermatocytes of the testes and oocytes of the ovaries in Cf-Piwil knockdown scallops, respectively, indicating germ cells at earlier development stages gradually died after earlier accumulation In addition, small numbers of spermatogonia and spermatids existed despite a great reduction of spermatocytes also demonstrated that spermatogenesis was blocked in spermatocytes. All these results suggest that $C f$-Piwil plays an important role in $C$. farreri gametogenesis.

\section{References}

Carmell MA, Girard A, Hj VDK, Bourc'His D, Bestor TH, de Rooij DG, and Hannon GJ. 2007.MIWI2 is essential for spermatogenesis and repression of transposons in the mouse male germline. Dev Cell 12:503-514.

Cerutti L, Mian N and Bateman A. 2000.Domains in gene silencing and cell differentiation proteins: the novel PAZ domain and redefinition of the Piwi domain. Trends Biochem Sci $25: 481-482$.

Chen R, Chang G, Zhang Y, Dai A, Ma T, Li J, Zhai F, Hua D, Xia M, and Chen G. 2012. Cloning of the quail PIWI gene and characterization of PIWI binding to small RNAs. PloS One 7:e51724.

Chomczynski P and Sacchi N. 1987. Single-step method of RNA isolation by acid guanidinium thiocyanate-phenol-chloroform extraction. Anal biochem 162: 156-159.

Cox DN, Chao A, Baker J, Chang L, Qiao D, and Lin H. 1998.A novel class of evolutionarily conserved genes defined by piwi are essential for stem cell self-renewal. Genes Dev $12: 3715-3727$.

Cox DN, Chao A and Lin H. 2000. piwi encodes a nucleoplasmic factor whose activity modulates the number and division rate of germline stem cells. Development 127:503-514. 
309

310

311

312

313

314

315

316

317

318

319

320

321

322

323

324

325

326

327

328

329

330

331

332

333

334

335

De MK, Pfister D, Kuales G, Egger B, Salvenmoser W, Willems M, Steger J, Fauster K, Micura R, and Borgonie G. 2009.Stem cells are differentially regulated during development, regeneration and homeostasis in flatworms. Dev Biol 334:198-212.

Deng W and Lin H. 2002. miwi, a murine homolog of piwi, encodes a cytoplasmic protein essential for spermatogenesis. Dev Cell 2:819-830.

Houwing S, Berezikov E, and Ketting RF. 2008.Zili is required for germ cell differentiation and meiosis in zebrafish. Embo J 27:2702-2711.

Houwing S, Kamminga LM, Berezikov E, Cronembold D, Girard A, Van dEH, Filippov DV, Blaser H, Raz E, and Moens CB. 2007. A role for Piwi and piRNAs in germ cell maintenance and transposon silencing in zebrafish. Cell 129:69-82.

Koichiro T, Peterson D, Peterson N, Stecher G, Nei M, and Kumar S. 2011. MEGA5: molecular evolutionary genetics analysis using likelihood, distance, and parsimony methods. Mol Biol Evol 28: 2731-2739.

Kuramochi-Miyagawa S, Kimura T, Ijiri TW, Isobe T, Asada N, Fujita Y, Ikawa M, Iwai N, Okabe M, and Deng W. 2004. Mili, a mammalian member of piwi family gene, is essential for spermatogenesis. Development 131:839-849.

Lau NC, Lim LP, Weinstein EG, and Bartel DP. 2001. An abundant class of tiny RNAs with probable regulatory roles in Caenorhabditis elegans. Science 294:858-862.

Li M, Hong N, Gui J, and Hong Y. 2012. Medaka piwi is Essential for Primordial Germ Cell Migration. Curr Mol Med 12:1040-1049.

Lin H and Spradling AC. 1997. A novel group of pumilio mutations affects the asymmetric division of germline stem cells in the Drosophila ovary. Development 124:2463-2476.

Lingel A, Simon B, Izaurralde E, and Sattler M. 2003. Structure and nucleic-acid binding of the Drosophila Argonaute 2 PAZ domain. Nature 426:465-469.

Liu J, Carmell MA, Rivas FV, Marsden CG, Thomson JM, Song JJ, Hammond SM, Joshuator L, and Hannon GJ. 2004. Argonaute2 is the catalytic engine of mammalian RNAi. Science 305:1437-1441. 
336

337

338

339

340

341

342

343

344

345

346

347

348

349

350

351

352

353

354

355

356

357

358

359

360

361

362

Liu J, Zhang Z, Zhang L, Liu X, Yang D, and Ma X. 2014.Variations of estradiol-17 $\beta$ and testosterone levels correlated with gametogenesis in the gonad of Zhikong scallop (Chlamys farreri) during annual reproductive cycle. Can J Zool. 92: 195-204.

Liu XL, Zhang ZF, Shao MY, Liu JG, and Muhammad F. 2012. Sexually dimorphic expression of foxl2 during gametogenesis in scallop Chlamys farreri, conserved with vertebrates. Dev Genes Evol 222:279-286.

Megosh HB, Cox DN, Campbell C, and Lin H. 2006. The role of PIWI and the miRNA machinery in Drosophila germline determination. Curr Biol 16:1884-1894.

Palakodeti D, Smielewska M, Lu YC, Yeo GW, and Graveley BR. 2008. The PIWI proteins SMEDWI-2 and SMEDWI-3 are required for stem cell function and piRNA expression in planarians. RNA 14:1174-1186.

Reddien PW, Oviedo NJ, Jennings JR, Jenkin JC, and Sánchez AA. 2005. SMEDWI-2 is a PIWI-like protein that regulates planarian stem cells. Science 310:1327-1330.

Rossi L; Salvetti A; Lena A; Batistoni R; Deri P; Pugliesi C; Loreti E; Gremigni V. 2006. DjPiwi-1, a member of the PAZ-Piwi gene family, defines a subpopulation of planarian stem cells. Dev Genes Evol 216:335-346.

Sasaki T, Shiohama A, Minoshima S, and Shimizu N. 2003. Identification of eight members of the Argonaute family in the human genome. Genomics 82:323-330.

Song JJ, Smith SK, Hannon GJ, and Joshuator L. 2004. Crystal structure of Argonaute and its implications for RISC slicer activity. Science 305:1434-1437.

Tatsuke T, Zhu L, Li Z, Mitsunobu H, Yoshimura K, Mon H, Lee JM, and Kusakabe T. 2014. Roles of Piwi proteins in transcriptional regulation mediated by HP1s in cultured silkworm cells. PLoS One 9:e92313.

Wang G and Reinke V. 2008. A C. elegans Piwi, PRG-1, regulates 21U-RNAs during spermatogenesis. Curr Biol 18:861-867.

Wang S, Hou R, Bao Z, Du H, He Y, Su H, Zhang Y, Fu X, Jiao W, and Li Y. 2013. Transcriptome Sequencing of Zhikong Scallop (Chlamys farreri) and Comparative 
363 Transcriptomic Analysis with Yesso Scallop (Patinopecten yessoensis). PLos One 8:

$364 \quad$ e63927.

365 Yan KS, Yan S, Farooq A, Han A, Zeng L, and Zhou MM. 2003. Structure and conserved RNA 366 binding of the PAZ domain. Nature 426:469-474.

367 Yano A, Suzuki K, and Yoshizaki G. 2008.Flow-cytometric isolation of testicular germ cells 368 from rainbow trout (Oncorhynchus mykiss) carrying the green fluorescent protein gene 369 driven by trout vasa regulatory regions. Biol Reprod 78:151-158. 


\section{Figure 1}

Phylogenetic analysis of Piwil among various species based on the multiple sequence alignment.

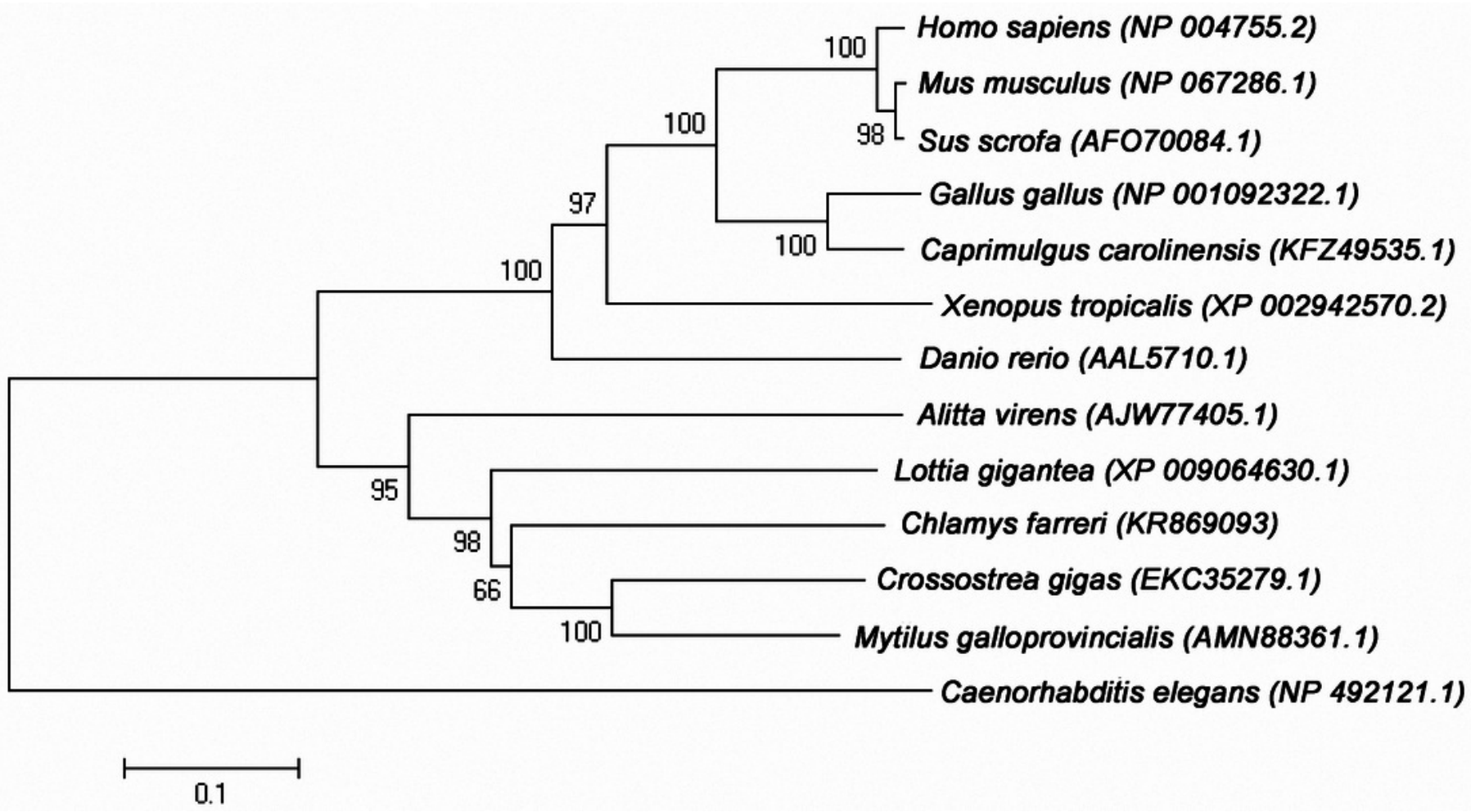




\section{Figure 2}

Relative abundance and location of Cf-Piwi1 mRNA in C. farreri gonads.

Relative abundance of Cf-Piwi1 mRNA detected by qRT-PCR in I) ovary and II) testis. The expression level in gonads at the resting stage was set as 1.00; Values are the mean \pm SEM; $\mathrm{n}=3$; Different letters indicate statistically significant differences $(P<0.05)$. III). Location of Cf-Piwil mRNA detected by tissue ISH. Negative ISH using a sense probe (b0, d0, g0). Positive signal from the anti-sense probe is stained in dark blue. (a), (b), (c) and (d), Ovaries at the resting, proliferative, growing, and mature stage, respectively; (e), (f), (g) and (h), Testes at the resting, proliferative, growing, and mature, respectively; (i), a different sight of the same section of (h) under microscope. ISC, Intragonadal somatic cell; Moc, Mature oocyte; Og, Oogonium; Oc, Oocyte; Sg, Spermatogonium; Sc, Spermatocyte; St, Spermatid; Sz, Spermatozoon. Magnification: Bar is $20 \mu \mathrm{m}$.

I )

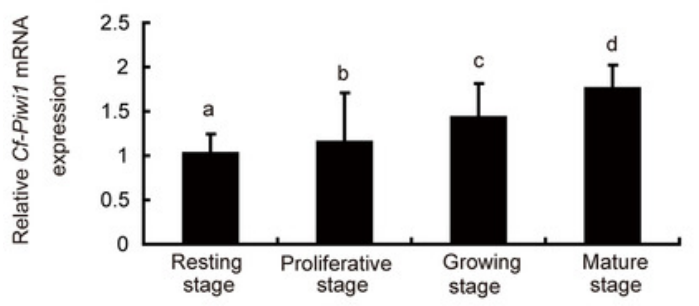

II )

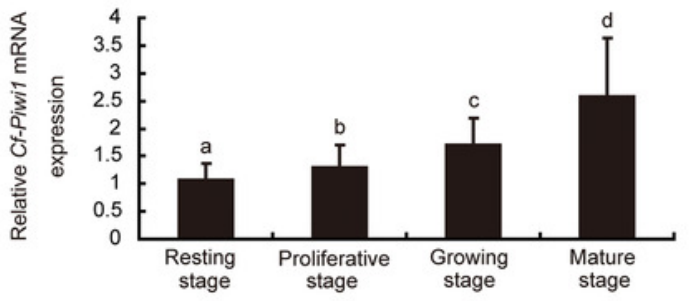

III)
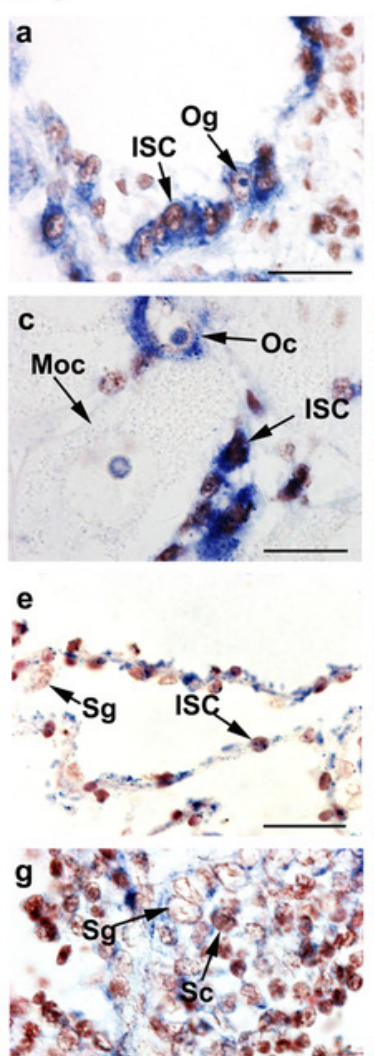

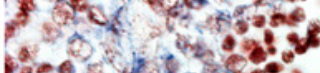
12 May 2017)
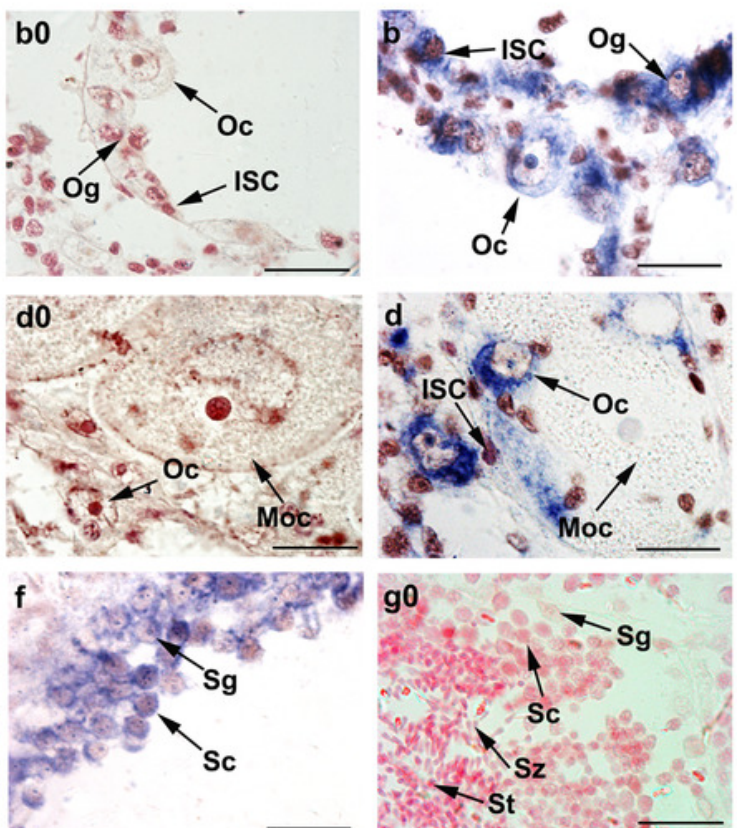
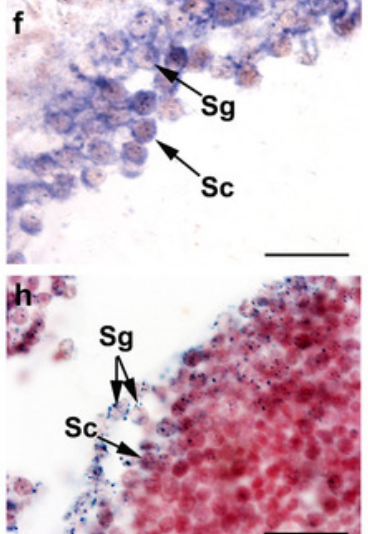

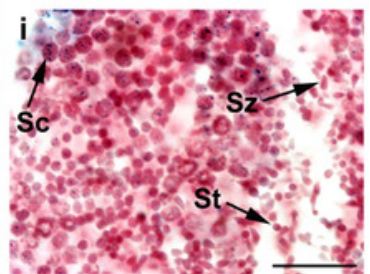




\section{Figure 3}

Expression of Cf-Piwi1 mRNA and histology of scallop gonads after RNAi.

Relative experiment level of Cf-Piwi1 mRNA detected by qRT-PCR in I) ovary and II) testis on 10th day after RNAi. The expression level in gonads of the blank group was set as 1.00;

Values are the mean $\pm S E M ; n=5$ in the testes; $n=3$ in the ovaries; Different letters indicate statistically significant differences $(P<0.05)$. III). Histological observation of scallop gonads on 10th day after RNAi. (a) and (d), ovary in the blank group; (b) and (e), ovary in the PBS group; (c) and (f), ovary in the dsRNA group; $(\mathrm{g})$ and (j), testis in the blank group; $(\mathrm{h})$ and (k), testis in the PBS group; (i) and (I), testis in the dsRNA group. Dc, Darkly stained cell; Og, Oogonium; Oc, Oocyte; Sc, Spermatocyte; Sg, Spermatogonium; St, Spermatid; Sz, Spermatozoon. Magnification: Bar is $40 \mu \mathrm{m}$ for (a), (b), (c), (g), (h) and (i); Bar is $20 \mu \mathrm{m}$ for (d), (e), (f), (j), (k) and (I).

I )

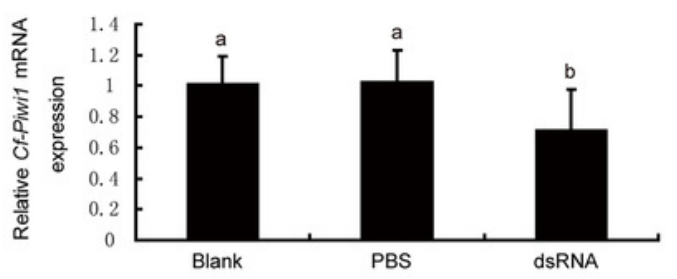

II)

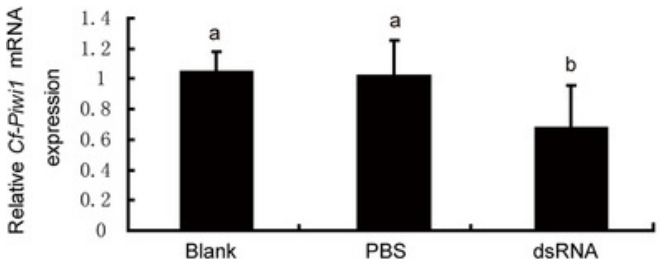

III)
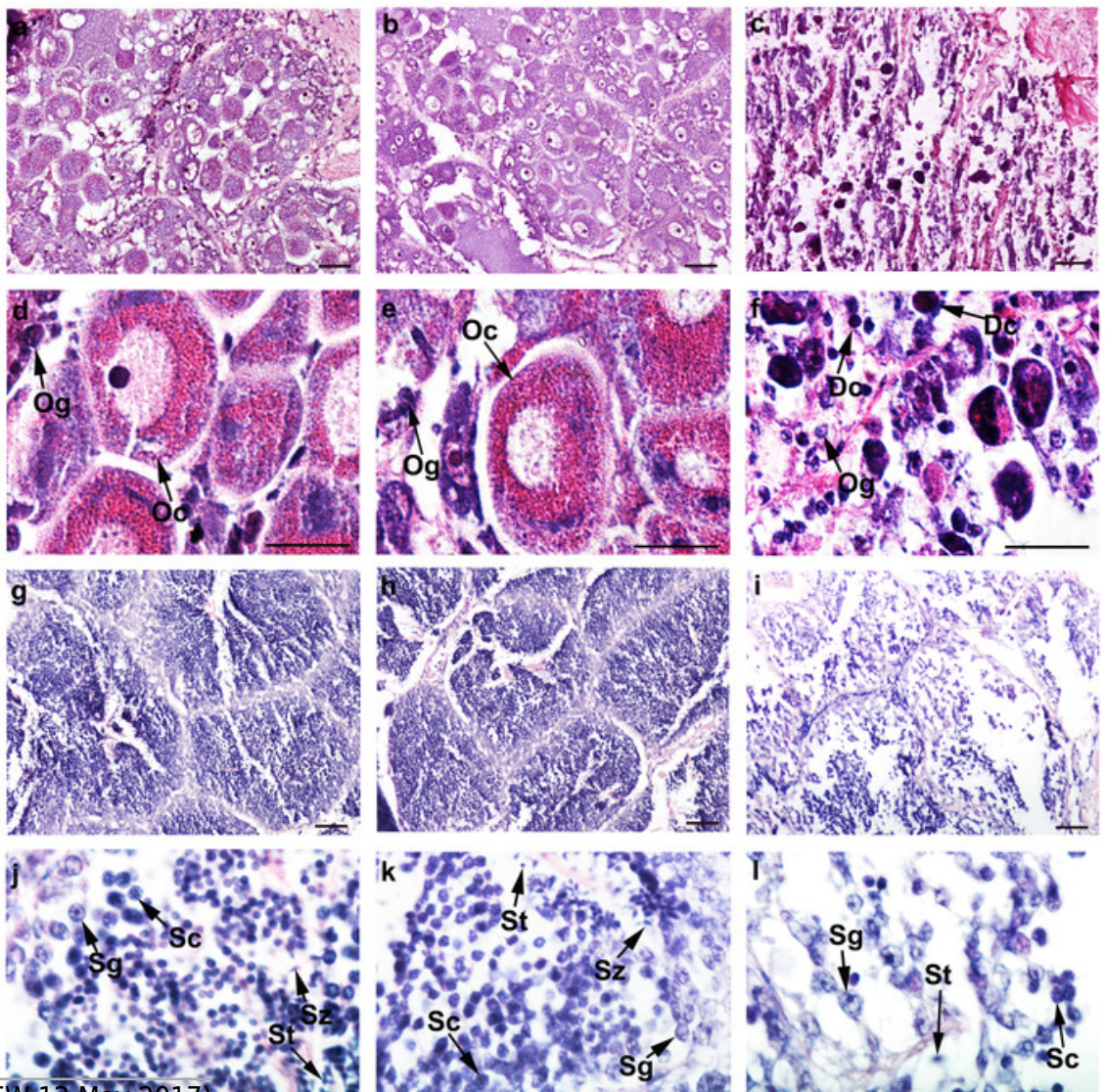


\section{Figure 4}

Quantification of the germ cells in scallop gonads after Cf-Piwi1 knockdown and cell apoptosis analysis.

Quantification of germ cells in I) ovary and II) testis and cell apoptosis detection III) on 10th day after RNAi. (a) and (d), ovary in the blank group; (b) and (e), ovary in the PBS group; (c) and (f), ovary in the dsRNA group; ( $g$ ) and (j), testis in the blank group; (h) and (k), testis in the PBS group; (i) and (I), testis in the dsRNA group. Apo, apoptosis cell; ISC, Intragonadal somatic cell; Moc, Mature oocyte; Og, Oogonium; Oc, Oocyte; Sg, Spermatogonium; Sc, Spermatocyte; St, Spermatid; Sz, Spermatozoon. The values are the mean $\pm S E M ; n=5$ in both testes and ovaries; different letters indicate statistically significant differences $(P<$ 0.05). Magnification: Bar is $40 \mu \mathrm{m}$ for (a), (b), (c), (g), (h) and (i); Bar is $20 \mu \mathrm{m}$ for (d), (e), (f), (j), (k) and (I).

I )

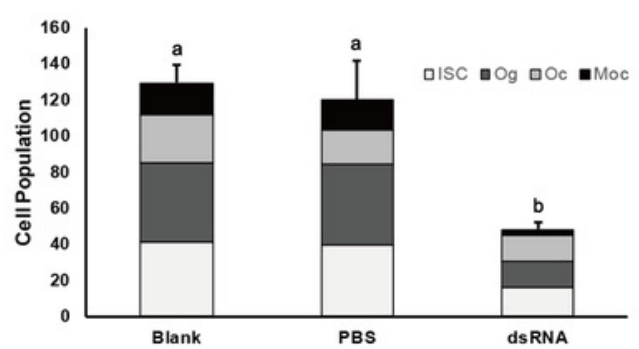

II )

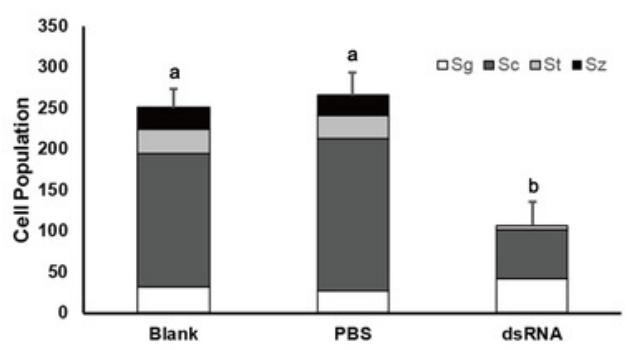

III)
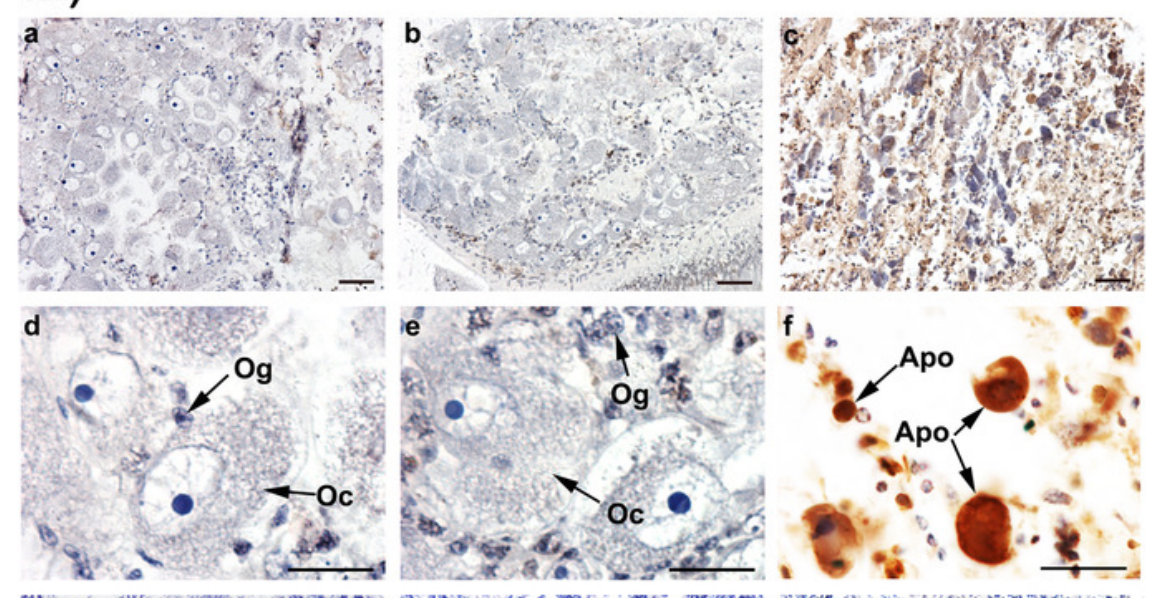

g
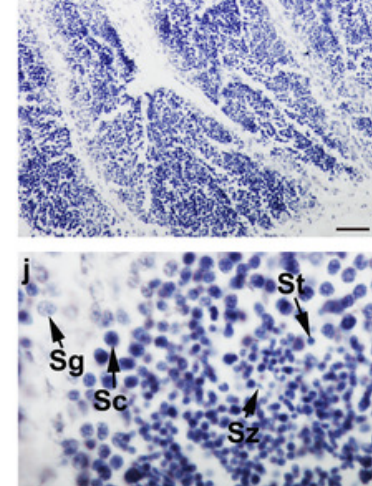

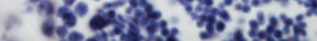
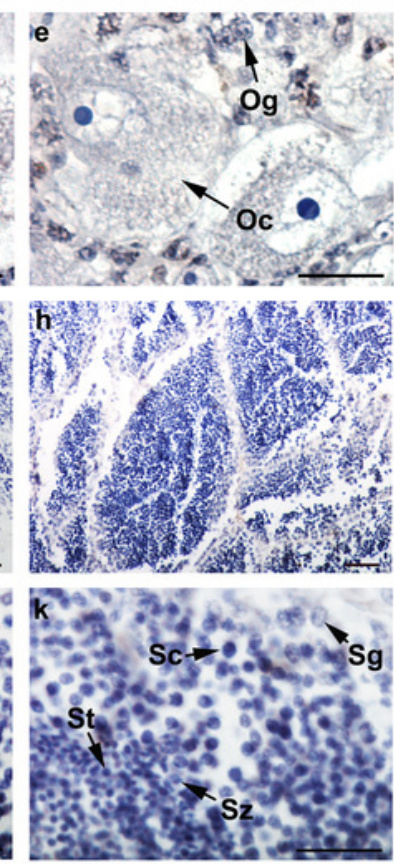

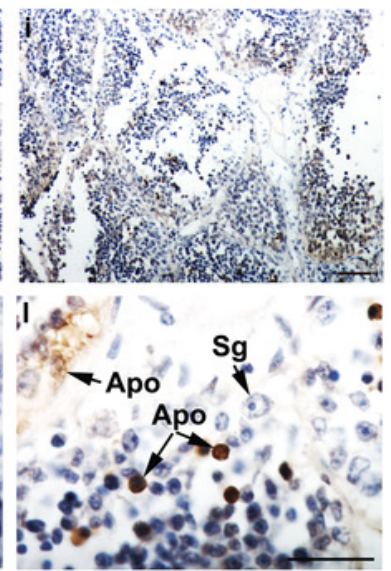

